\title{
Beten aus Gewohnheit?
}

\section{Einwendung gegen eine Verwendung von Merleau-Pontys Leibbegriff zur Bestimmung des Gebetsphänomens}

Marcel Egli

Der Einwand gegen die Verwendung des Leibbegriffs im Zusammenhang des Gebetsthemas richtet sich gegen einen an MerleauPonty orientierten Leibbegriff. Dieser Leibbegriff besagt, dass Leib im Sinne des leiblichen Verhaltens zu verstehen ist. Und falls dieses Verhalten sich in bestimmten Situationen etabliert, sei die erreichte Routine als >verleiblichtes Verstehen bestimmen. Gegen die Auffassung, dieser Leibbegriff sei auch im Zusammenhang des Gebetsphänomens sinnvoll, richtet sich im Folgenden die Argumentation. Die vorgetragene Kritik an diesem Leibbegriff profiliert sich zwar an Merleau-Pontys Bestimmung von \Leib<. Anderen, im weitesten Sinne gleichfalls anthropologischen Leibkonzepten, gilt dieser Einwand aber ebenso; sie sind im $\mathrm{Zu}-$ sammenhang des Gebetsthemas wenn auch nicht ungültig, so doch nicht exklusiv. Sie können nicht unwidersprochen Geltung in Anspruch nehmen.

Merleau-Pontys Leibbegriff wird im Folgenden so weit dargestellt und behandelt, als es für das Verständnis seines Konzepts des leiblichen Verstehens notwendig ist. Dieses Konzept besagt, dass aus dem leiblichen Verstehen eine Gewohnheit entsteht. Wenn sich das Verstehen verleiblicht, so ist das, was verstanden worden ist, zur Gewohnheit geworden. Merleau-Pontys Leibbegriff ist nun für viele Beschreibungen von Praktiken sinnvoll und erhellend; es gibt aber auch Beschreibungsweisen, bei denen sich dieser Begriff nicht nahelegt. So ist es beispielsweise weder selbstverständlich noch voraussetzungslos angemessen, das Gebet eben als eine Gewohnheit zu bestimmen. Denn es setzt ein Leibkonzept voraus, das dem Gebetsphänomen nicht zwingend gerecht wird. Die möglichen Einwände gegen dieses Konzept betreffen das mit ihm vorausgesetzte Wirklichkeitsverständnis. Es stellt sich die Frage, ob die für das Gebet konstitutiven Relationen (zu denen die Beziehungen zwischen Gebet, Betendem und Angebetetem zählen) sinnvoll berücksichtigt werden. $\mathrm{Zu}$ klären ist, ob es sachgemäß ist anzunehmen, dass der Betende selber oder auch jemand, der den Gebetsvorgang beobachtet, um 
ihn zu bestimmen, sich, durch Einüben und Angewöhnen, an den Vorgang und an das im Gebet Intendierte gewöhnen kann.

Diese Anfragen betreffen die Überzeugung, im Gebet würde sich etwas zu etwas entwickeln (das Gebet zu einem Verstehen). Sie gründen ihrerseits in der Überzeugung, dass sich das Gebetsphänomen rechtmäßig dann erschließt, wenn der Fokus auf den Vorgang des Betens selbst gerichtet wird. In diesem Zugang zum Gebetsphänomen erhält der Leib einen anderen Stellenwert, als er in Merleau-Pontys Konzept vorgesehen ist. Der Leib ist dann nicht das Mittel, mit dem das Gebet vollzogen wird, sondern der Vorgang des Gebets selbst. In dieser Sichtweise dient der betende Leib dem Beten selbst. Diese Bestimmung des Leibes folgt nicht einer dem Betvorgang externen Beschreibungsweise. Entsprechend wird mit 'Leib nicht der am Vorgang beteiligte Handlungsträger bestimmt, sondern das leibliche Hervorgebrachtwerden des betenden Ich.

Konkret geht es im Folgenden darum, der Bestimmung des Leibes als eines $>$ Vehikels $<$ (Merleau-Ponty) die Bestimmung des Leibes als eines Vorganges der `Leibung`(Jean-Luc Marion) gegenüberzustellen. Zur Darstellung dieser Opposition wird erörtert, von welchen Voraussetzungen sich die Bestimmung des Gebets als eines sverleiblichten Verstehens` leiten lässt und zu welchem Ergebnis sie gelangt. Dieser wird die andere Zugangsweise gegenübergestellt. Durch dieses Gegenüberstellen wird die Bestimmung des Gebets als >verleiblichtes Verstehen v verortet. Dieses Verorten ist ein Widersprechen und Opponieren. Die beiden Zugangsweisen finden ihren jeweiligen Ort nämlich nicht in einer gemeinsamen Ordnung, sondern mittels eines Widersprechens von der jeweils ganz anderen Position her. Gegenüber der Bestimmung des Gebets als ıverleiblichtes Verstehen<, und damit als Gewohnheit, wird die Position vertreten, das Gebet sei eine Nicht-Gewohnheit, bei der der Leib nicht als der Leib des Betenden $\mathrm{zu}$ bestimmen ist, sondern als der einer anderen leiblich wirksamen >Instanz , die den Betenden erst zum Betenden macht. Eine Vermittlung der beiden Zugangsweisen ist nicht möglich. Für den Betenden, der sein eigenes Beten verstehen will, wäre das Gebet dann zu bestimmen nicht als ein Verstehen von etwas, sondern als ein anfängliches Selbstverstehen, das einem vollkommenen Nichtund Nichts-Verstehenkönnen folgt.

Für die dargestellte Problematisierung des Leibbegriffs leitend ist die Überzeugung, dass sich nicht von selbst erklärt, was \Leib bezeichnet, sondern dass jeder Leibbegriff bedingt ist von einem bestimmtem Gottesbegriff und einem entsprechenden Wirklichkeitsverständnis. Die umrissene Kritik fokussiert auf zwei Relate des 
Gebetsvorgangs: die `Instanz` Gott als die Größe, auf die das Gebet bezogen ist, sowie der betende Leib als die Größe, in und an der das sich auf Gott beziehende Gebet als Gebet erscheint. Entscheidend dabei ist, wie die Relation dieser Relate verstanden wird.

Die Darstellung folgt dieser Gliederung: Vorab ist zu klären, was in der folgenden Untersuchung überhaupt > Verstehen heißt (1.). Dann wird beschrieben, mit welchen Problemen sich das Bestimmen des Gebetsphänomens prima facie konfrontiert sieht und wie mit diesen umzugehen ist (2.). Es folgt die Darstellung des Leibbegriffs von Merleau-Ponty (3.). Auf die Kritik an dieser Position (4.) folgt die Darstellung des Begriffs der `Leibungく von Jean-Luc Marion (5.), um zum Schluss (6.) diesen Ansatz konstruktiv aufzunehmen und - die Kritik an Merleau-Pontys Leibbegriff fortsetzend - in explizit theologischer Sprache auf das Gebetsphänomen hin auszulegen.

\section{Klärungen}

\section{`Verstehen}

Damit die gegebene Fragestellung deutlich wird, ist eine begriffliche Klärung unerlässlich. D.h., im Verfahren, das Gebet als sverleiblichtes Verstehen zu verstehen, ist der Begriff `(verleiblichtes) Verstehen ‘ von dem Vollzug und dem Resultat des Verstehens dieses Verstehens zu unterscheiden. Denn Verstehen ist dabei auf zwei Ebenen im Spiel: 1) Verstehen im Sinne von ssich mit etwas auskennen', ssich mit etwas vertraut machen «, ssich an etwas gewöhnen<; 2) Verstehen im Sinne von retwas als etwas bestimmen. Für die erste Verwendungsweise wird im Folgenden der Begriff , Verstehen « verwendet, für die zweite der Begriff >Bestimmen . Während im ersten Fall das Sich-Gewöhnenan-etwas gemeint ist, geht es im zweiten Fall darum, ein einzelnes Phänomen in einem allgemeinen Verstehenszusammenhang zu verorten, um es als etwas zu verstehen. Wenn in der Untersuchung des Gebetsphänomens das Gebet also als ein sverleiblichtes Verstehen bestimmt wird, so geht es dabei um eine Untersuchung, durch die der Vorgang, bei dem ein Können und eine Fertigkeit erlangt wird, als das Erlangen eines Könnens bzw. einer Fertigkeit oder eben als eine Gewohnheit bestimmt wird. Der Einzelfall des Gebetsvorgangs wird als Fall der Gruppe der Gewohnheiten bezeichnet.

Der Versuch nun, das Gebet als verleiblichtes Verstehen $b z w$. als , Gewohnheit zu bestimmen, steht vor der Aufgabe, beide genannten 
Weisen von Verstehen zu integrieren. Er hat die beiden oben genannten Verstehensaspekte zusammenzufassen: 1) den Vorgang des Sich-auf-etwas-Verstehens und 2) das Bestimmen dieses Sich-auf-etwasVerstehens. Nicht nur das Bestimmen ist ein Verstehen, sondern auch das Erlangen der Fertigkeit, beispielsweise beten zu können. Der Versuch hat einerseits die Innerlichkeit und damit einhergehende spezifische Unsichtbarkeit des Gebets als Gewohnheit zu beachten; und es gilt darüber hinaus, dieses nicht eindeutige, sondern stets mehreren Verständnissen offene Phänomen zu bestimmen und in einem eigenen Konzept kohärent zu beschreiben. Das resultierende Gebetskonzept dient dann dem Bestimmen eines im Leib beheimateten Verstehens. Anders gesagt: Das Bestimmen, dem der Status des Allgemeinen des Verstandes eignet, versucht die Verstehensbemühung zu bestimmen, die ihren Ort im und am Besonderen eines einzelnen Leibes hat. Es geht zweimal um einen Verstehensvorgang: um das Bestimmen eines einzelnen Vorgangs durch die Allgemeinheit von Begriffen sowie das Selbstverstehen eines Einzelnen.

\section{Phänomenalität des Gebets}

Während hinsichtlich der Fragestellung obige Erläuterung nötig war, ist eine Klärung auch in Bezug auf die Phänomenalität selbst, d.h. die Erscheinungsweise und den Ort eines leiblichen Vorgangs (z.B. eben des Gebets) unerlässlich. Zu klären ist die Weise, wie sich der Vorgang seinem Betrachter zeigt. Es muss geklärt sein, wie sich das Phänomen dem Vorhaben darbietet, das es als etwas bestimmt. Mit der Bezeichnung des Phänomens als eines 'verleiblichten Verstehens ist bereits eingegrenzt, wie es sich zeigen kann; mit dieser Bezeichnung ist eine bestimmte Phänomenalität des Phänomens gegeben. Es ist einerseits ein Phänomen, das leiblich ist. Es zeigt sich im, am und durch den Leib des am Vorgang Beteiligten. Wenn der Vorgang leiblich ist, so ist es ein innerlicher (leiblich-innerlicher) Vorgang. Denn ein verleiblichtes Verstehen ist primär ein Sich-selbst-auf-etwas-Verstehen. Mit der ergänzenden Bezeichnung als >Verstehen « aber ist nicht bloß dieser Bezug auf den am Vorgang Beteiligten selbst, der sich selbst auf etwas versteht, gemeint, sondern zudem eine bestimmte Rationalität des Vorgangs vorausgesetzt. Er ist nicht nur ein innerlicher Vorgang, sondern ebenfalls eine grundsätzlich auch anderen mögliche Verstehensbemühung. Er vollzieht sich auf eine rationale Weise, so dass er nicht nur für denjenigen, der ihn vollzieht, sondern auch für andere, die seine Vernunft teilen, nachvollziehbar ist. Wenn der Vorgang ein Verstehen ist, so ist er nicht nur ein verborgener, sondern stets auch 
ein rationaler (nicht nur leibgebundener, sondern auch vernünftiger) Vorgang des Menschen. Diese beiden Eigenschaften des leiblichen Verhaltens sind konstitutiv. Wer sich verhält (indem er z.B. betet), tut dies leiblich, also bei sich und für sich selbst; er tut dies aber auch auf eine allgemein-menschliche Weise.

Trotz der Allgemeinheit dieser leiblichen Verstehensbemühung, bedeutet die Leiblichkeit dennoch eine Gebundenheit an einen bestimmten Ort (eine Position). Der leibgebundene Vorgang lässt sich nicht anderswohin delegieren. Wenn jemand z.B. selbst nicht betet, betet niemand anders für ihn sein Gebet. Mit der Leiblichkeit ist in Bezug auf den faktischen Vorgang ein Allgemeinheitscharakter, der beim verstehenden Bestimmen und dem analogen Mit- oder Nachvollzug gegeben ist, ausgeschlossen. In seiner Leiblichkeit ist das Gebet die Handlung eines unvertretbar Einzelnen, die sogar für jene Menschen, mit denen der Betende seine Vernunft teilt, ein fremder Vorgang ist und bleibt. So wie sich der Gebetsvollzug mit niemandem teilen lässt, lässt sich vom Gebet auch nicht alles vermitteln. Das Gebet als das leibliche Verhalten eines Einzelnen entzieht und verbirgt sich. Verborgen bleibt dabei nicht nur - wie oben erläutert - das, wodurch und womit leiblich-innerlich gebetet wird. Es entzieht sich darüber hinaus auch, was angebetet wird. Das, worauf sich das Gebet richtet, tritt im beobachtenden Beschreiben des Gebetsphänomens nicht auf. Diese Stelle bleibt leer.

\section{Das Gebetsphänomen bestimmen}

Das Gebet, konzipiert als 'verleiblichtes Verstehen', ist also ein leibliches und damit innerliches Geschehen, hat aber zugleich einen rationalen Charakter, insofern es einen Gehalt aufweist, der sich nicht nur für einen selbst, sondern auch für andere, die beten können, verständlich machen lässt. Das Gebet ist dem Verstehen entzogen, aber auch als ein vernünftiger und rationalerVorgang dem Verstehen zugänglich. Es ist ein Phänomen, das sowohl sich verbirgt, als auch in gewisser Weise zu Tage tritt. Es verbirgt sich, indem es sich im Inneren des Betenden abspielt, ist aber auch Sprache und zeigt sich, indem es als Gebetsformel, Geste oder Ritual eine Form annimmt, die auch anderen zugänglich ist und von ihnen adaptiert werden kann. 


\section{Problem}

Das Problem, dem sich das Konzept des iverleiblichten Verstehens stellt, lässt sich damit ganz grob wie folgt fassen: Es gibt keine Formen des Gebets, in denen sich der Gebetsvorgang in allen Aspekten hinreichend erschließt. Selbst im Fall der Anbetung nicht. So zeigt z.B. das angebetete Jesuskind in der Darstellung der >Anbetung der Königer nicht alles von sich, sondern verbirgt seine Identität als Messias. Zeigte sich in dieser Situation alles offen, wäre es keine Anbetungssituation. In Fällen, wo sich etwas eindeutig als Intendiertes zeigt, handelte es sich bloß um einen Akt der Bewunderung: Der Jesus wird dann als neuer Herrscher, als niedlicher Säugling usf. bewundert. Bloße Bewunderung ist jedoch keine Anbetung. Die Messianität des Jesuskindes bleibt verborgen, weil nur die Verborgenheit die anbetungswürdige Messianität anzeigt.

In Anbetung und Gebet ist nicht nur das, worauf sich das Gebet richtet, für den, der nicht betet, nicht wirklich und gilt als bloß gedacht, eingebildet oder krankheitsbedingt halluziniert. Sondern es zeigt sich ihm auch nicht alles vom Gebet; dem bloß betrachtenden Dritten bleibt einiges zwingend verborgen. Ohne das Sich-Entziehen im Gebetsphänomen gibt es für den Betrachter kein Verstehen des Gebets. Dem Betrachter erschließt sich das Gebet entweder gar nicht oder, wenn doch, nur unzureichend. Als liturgisch gestaltete Handlung einer Gruppe oder eines Einzelnen ist es als Formel, Geste oder Ritual zwar für den Betrachter vernehmbar und beschreibbar (wenn auch nicht darin, worauf es sich richtet). Als stilles Gebet wird es ebenso nur unzureichend entschlüsselt. Und das sglaubende Gerichtetsein^ des Betenden schließlich bleibt ihm vollständig verborgen. Dennoch gilt jede dieser Formen als Gebet.

Aus dieser Problembestimmung folgen Untersuchungsfragen wie: Unter welchen Umständen und mit welchen Mitteln kann ein Vorgang dennoch als Gebet identifiziert und beschrieben werden?: in der Beteiligtenperspektive?, im separaten Nachvollzug? usf. Und als was soll es beschrieben werden?: als Widerfahrnis?, als umwälzendes Ereignis? usf.

\section{Methode und deren Problematik}

Die These wird unten sein, dass die Beschreibung des Gebets nur dann sinnvoll sei, wenn sie den Blick vom Betenden abwendet und dem Woraufhin des Gebets eine für das Gebet konstitutive Rolle zuweist. In dieser Perspektive vergrößert sich das Spektrum der Fragen, die die Untersuchung leiten können: Es ist vielleicht nicht nur 
möglich, in der Beteiligtenperspektive oder im separaten Nachvollzug etwas als Gebet zu identifizieren und zu beschreiben. Sondern vielleicht zeigt sich das Gebet auch gar nicht. Und es ist vielleicht nicht nur möglich, das Gebet als ein Widerfahrnis oder eine Umwälzung $\mathrm{zu}$ bestimmen. Sondern vielleicht ist es auch ein Geschehen in und an einem Anderen für mich. Und es wird die zentrale Frage schließlich ausdrücklich zu beantworten sein, ob die Verborgenheit des Gebets an der (prinzipiellen, d.h. von Gott selbst bedingten) Verborgenheit des Adressaten des Gebets (Gott) liegt, an der Schwäche des Betenden, die Gott-Mensch-Relation hinreichend zum Ausdruck zu bringen oder am Unvermögen oder Unwillen des Betrachters des Gebets, recht zu sehen. Die Wahl derVariante zur Beschreibung des spezifischen Verborgenseins des Gebetsvorgangs und seines Kontexts entscheidet sich wohl am verwendeten Gottesbegriff und damit daran, von welchem Wirklichkeitsverständnis sich die Verstehensbemühung leiten lässt - hinsichtlich nicht nur Gottes, sondern auch der Welt und des Menschen. In dieser Wahl zeigt sich insbesondere der leitende Leibbegriff.

\section{Der Leib nach Merleau-Ponty}

Merleau-Pontys Begriff des Leibes, dem im Folgenden die Kritik gilt, steht in Verbindung mit seinem Konzept von Wahrnehmung. Er widerspricht in seiner `Phänomenologie der Wahrnehmung`sowohl dem empiristischen als auch dem subjektivistischen Konzept von Wahrnehmung. Das Wahrgenommene ist so wenig als das zu bestimmen, was an Lauten hör- oder an Gesten sichtbar ist (Empirismus), wie als das, wozu ein Subjekt aufgrund seiner Anlage in der Lage sein soll (Subjektivismus). Der Leib ist für Merleau-Ponty sowohl die Instanz, die wahrnimmt, als auch jene, wodurch die Wahrnehmung vollzogen wird. Diese Unmittelbarkeit von Leib und Welt ist für Merleau-Pontys Leibverständnis charakteristisch.

Die Weise, wie die Welt als Wirklichkeit wahrzunehmen ist, d.h. die Einstellung zur Welt, ist nach Merleau-Ponty keine natürliche, sondern eine transzendentale. Was mir als Welt gegeben ist, ist nicht unabhängig von mir da, sondern entsteht mit meiner Wahrnehmung der Welt ${ }^{1}$. Diese Wahrnehmung bezieht sich nicht auf etwas ihr Vorgegebenes, sondern ist Bedingung der Möglichkeit des Wahrgenom-

\footnotetext{
1 Vgl. Bernhard Waldenfels, Phänomenologie in Frankreich, Frankfurt a.M. 1998, 31: "Im Übergang von der natürlichen zur transzendentalen Einstellung zeigt sich keine andere Welt, aber die Welt zeigt sich anders, unfertig, entstehend, erschlossen
} 
menen $^{2}$. Bezeichnet >Welt die Gesamtheit dessen, was mir gegeben sein kann, so ist, was immer mir gegeben ist, nicht für sich da, sondern dadurch, dass ich es wahrnehme. Wahrnehmen ist im Anschluss an Merleau-Ponty zudem zu bestimmen sowohl als eine Handlung, die der Wahrnehmende sich als mentale, sprachliche, gestische usf. Form angeeignet hat, als auch als das, was er davon ablehnt und dem er sich entzieht. Es geht um die Übernahme einer überlieferten Form, in der diese Form angeeignet wird, indem sie als kontingente fremde Form abgelehnt und als eigene und damit notwendige Form angenommen wird. Der fremden Form verpflichtet, befreit sich der, der wahrnimmt, dennoch von ihr, indem er sie sich aneignet und in seinen Besitz nimmt.

Diese aneignende Übernahme ist ein leibliches Verhalten, insofern nämlich die Übernahme durch den Leib vollzogen wird. Im Leib fallen zusammen der, der übernimmt, und das, was übernommen wird. Im Leib gibt es beide Größen, Innen- und Außenwelt. Als diese Einheit ist der Leib die Voraussetzung dafür, dass der Übernahmevorgang sich durch und für ein Subjekt vollzieht. Der Leib ist "weder Ding noch Bewußtsein, sondern eine unentbehrliche Vorgabe meiner selbst $\aleph^{4}$. Als leibliches Verhalten erst wird ein Vorgang zu einer Handlung eines bestimmten Subjekts. Als leibliches ist es unvertretbar das Verhalten dieses Subjekts. Das Subjekt sagt: Ich bin selbst dieser Leib, "zumindest in dem Maße, in dem ich einen Erwerb mein eigen nenne, und umgekehrt ist mein Leib gleichsam ein natürliches Subjekt, ein vorläufiger Entwurf meines Seins im ganzen $\ll^{5}$.

Merleau-Ponty sucht eine Alternative zu Empirismus und Subjektivismus. In seiner `Phänomenologie der Wahrnehmung` vertritt er

und verschlossen zugleich, mit einem Sinn in statu nascendi, wie Merleau-Ponty sich wiederholt ausdrückt."

2 Vgl. Lambert Wirsing, Merleau-Pontys Entdeckung der Wahrnehmung [Nachwort], in: Maurice Merleau-Ponty, Das Primat der Wahrnehmung, Frankfurt a.M. 2003, 94f: Leibliche Wahrnehmung bedingt die Perspektivität ohne selbst je Teil von ihr zu werden.

3 Vgl. Maurice Merleau-Ponty, Phänomenologie der Wahrnehmung [Übers. Rudolf Boehm], Berlin 1966, 203f: "Die menschliche Existenz nötigt uns, die gängigen Begriffe von Notwendigkeit und Kontingenz zu revidieren, da sie selbst die Verwandlung von Kontingenz in Notwendigkeit durch den Vollzug der Übernahme ist. Alles, was wir sind, sind wir auf Grund einer faktischen Situation, die wir uns zu eigen machen und unabläßlich verwandeln durch eine Art von Entzug, der gleichwohl nie zur unbedingten Freiheit wird."

4 Waldenfels, Phänomenologie in Frankreich, 167.

5 Merleau-Ponty, Phänomenologie der Wahrnehmung, 234.Vgl. Waldenfels, Phänomenologie in Frankreich, 167. 
die Überzeugung, dass Wahrnehmung leiblich zu verstehen ist. Der Leib ist weder auf natürlich-empirische, noch auf subjektive Weise als Grundlage von Wahrnehmung zu bestimmen. Der Leib ermöglicht vielmehr Wahrnehmendes und Wahrgenommenes überhaupt erst. Der Leib ist die transzendentale Bedingung der Möglichkeit von Wahrnehmung.

\section{Der Leib als \Vehikel des Zur-Welt-seinsı (Merleau-Ponty)}

"Der Leib ist das Vehikel des Zur-Welt-seins, und einen Leib haben, heißt für den Lebenden, sich einem bestimmten Milieu zugesellen, sich mit bestimmten Vorhaben identifizieren und darin beständig sich engagieren « ${ }^{6}$.

Der Leib ist für Merleau-Ponty Wahrnehmendes und Wahrgenommenes zugleich. Sein Anliegen ist es, den Leib nicht als bloßes Objekt der Wahrnehmung zu verstehen, sondern gleichwertig als die Instanz, die den Leib zu einem wahrnehmbaren Objekt macht. Ohne wahrnehmenden Leib gibt es keinen wahrgenommenen Leib. Den Ort des in dieser Weise doppelt bestimmten Leibes bezeichnet Merleau-Ponty als `Milieu $<$. In diesem Bereich hat der Leib jedoch nicht seinen festen Ort, sondern das `Milieu $<$ ist ein Ort, an dem etwas auf dynamische Weise geschieht: Darin kommt und geht die Existenz, bald körperlich sich zutragend, bald wieder persönlich handelnd ${ }^{7}$. Hätte der Leib einen festen Ort, wäre er ein Objekt in der Welt (wie die Dinge). So ließe er sich aber nicht eindeutig einer persönlichen Existenz zuweisen. Er wäre zwar lokalisierbar, aber niemand würde ihn als eigenen Körper identifizieren in der Weise, dass er in seinem Verhalten auf ihn zurückkommen und ihn sich selber zurechnen würde. Der Begriff des `Milieu < bringt zum Ausdruck, dass leibliches Verhalten in einer durch dieses Verhalten geschaffenen Konstellation stattfindet. Unabhängig von diesem tätigen leiblichen Verhalten selbst gibt es dieses >Milieu< nicht. Obwohl es stattfindet, hat es jedoch keine Geschichte. Obwohl es durch jemanden geschieht, ist es keine biographisch relevante Erfahrung. Der Leib dient nicht der Ortsbestimmung in der Welt, sondern ist die Weise, sich zu seiner Welt zu verhalten. Der Körper ist von Merleau-Ponty bestimmt als das , Vehikel des Zur-Welt-seins`. Damit wird die Situation des Körpers nicht in einem ihm fremden Ordnungsgefüge bestimmt, sondern

6 Merleau-Ponty, Phänomenologie der Wahrnehmung, 106.

7 Vgl. ebd., 113. 
seinen Aufgaben gegenüber, mit denen er zu tun hat und denen er aufgrund seiner Position verpflichtet ist.

Bezogen auf das Gebet ist somit das, worauf sich das Gebet richtet, erst dadurch da, dass es angebetet wird. Die Übernahme des Gebets durch einen Leib ist das Vehikel für das Angebetete. Das Gebet kann verstanden werden als Wahrnehmung. Wahrgenommen bzw. angebetet wird, woraufhin das Wahrnehmen bzw. das Beten ausgerichtet ist. Ohne Wahrnehmung kein Wahrgenommenes, ohne Beten kein Angebetetes. Und ohne Wahrnehmen bzw. ohne Beten kein Ort der Wahrnehmung und des Betens. Wahrnehmen wie Beten findet seine Statt im Vollzug. Dies ist der Grund dafür, dass sich dem Betrachter des Gebets keine Konstellation darbietet, die sich erschließt, insofern sich an ihr Ursache und Wirkung analysieren lassen. Angebetetes und Anbetung können nicht unterschieden werden. Selbst dem Betenden bleibt eine nicht betende Wahrnehmung dessen, was er anbetet, verschlossen. Folglich ist Beten weder nur das Werk dessen, was angebetet wird, noch nur dessen, der betet, sondern im Gebetsvorgang ko-operieren beide Instanzen.

Das, worin religiöse Erfahrung im `Milieu des Gebets Form annimmt, könnte als `Gebetsleib` bestimmt werden. Im >gebetsleiblichen Verhalten erschließt sich dem Betenden das Angebetete und darin - wie gleich zu zeigen ist - übernimmt der Betende tradierte religiöse Ausdrucksformen als seine eigenen. Durch dieses Verhalten ist er jedoch nicht zu bestimmen als Anhänger einer Gruppe, die bestimmte religiöse Formen pflegt und tradiert, sondern weiterhin als Betender, der für sich betet. Die Bestimmung des Betens als 'gebetsleibliches Verhalten beschreibt nicht eine durch Kategorisierung gewonnene Lokalisierung des Betenden, sondern seine Selbstidentifizierung. Als `Gebetsleib ist der Betende bestimmt als der Betende, der er betend selbst ist.

\section{Gewohnheit, sich gewöhnen}

Der Leib ist für Merleau-Ponty Bedingung der Möglichkeit von Wahrnehmung. Vorgänge, in die der Leib involviert ist, sind für den Wahrnehmenden grundlegend. Ohne Leib gibt es keine Wahrnehmung. Das Ergebnis des Wahrnehmens ist das, was sich aufgrund solchen leiblichen Verhaltens ergibt. Merleau-Ponty nennt das, was sich aus vollzogener Wahrnehmung ergibt, $>$ Gewohnheit .

»Verstehen heißt, die Übereinstimmung erfahren zwischen Intention und Vollzug, zwischen dem, worauf wir abzielen, und dem, was gegeben ist; 
und der Leib ist unsere Verankerung in der Welt" ". "Man sagt, der Leib habe verstanden und die Gewohnheit sei erlangt, wenn er von einer neuen Bedeutung sich hat durchdringen lassen, einen neuen Bedeutungskern sich angeeignet hat «?

Merleau-Ponty beschreibt erfolgtes verleiblichtes Verstehen als Gewohnheit. So hat sich beispielsweise der Blinde an die Handhabung seines Stockes gewöhnt, wenn er die Beschaffenheit seiner unmittelbaren Umgebung nicht mehr direkt mit seinem Körper ertastet, sondern mit demselben Erfolg mittels seines Stockes abtastet.Von dem Zeitpunkt an, da der Blinde sich auf die Verwendung des Stockes versteht, ist ihm dessen Handhabung in Fleisch und Blut übergegangen. Sein Stock hat für ihn die Funktion eines seiner Körperteile übernommen. Das Hantieren mit dem Blindenstock ist ihm zur Gewohnheit geworden.

Wenn nun das Gebet sich jemandem, d.h. seinem Denken, Sprechen und Handeln, inkorporiert, ist es ihm zu einer Gewohnheit geworden. Die Gebetsgewohnheit kommt dabei durch leibliches Verhalten zustande: Mittels des Gebets (als Text, Formel, Geste oder Ritual) wird gebetet (sprachliche Tätigkeit, Bewegung). Dieses leibliche Verhalten ist das Mittel, mit dem gebetet wird. Wenn sich der Betende an es gewöhnt, so ist er (als Leib) mit dem Gebet identisch geworden. Er ist zum Organ und Medium seines Gebets geworden. Er betet mit und durch sich selbst. Die aus der Tradition übernommenen Praktiken und Medien sind übernommen und angenommen worden. Diese Traditionsträger wurden vertraut und wurden angeeignet. Man gibt mit Erlangen der Gewohnheit nicht mehr etwas Fremdes wieder, sondern bringt Eigenes zum Ausdruck.

Merleau-Pontys Leibkonzeption hat ein bestimmtes Anliegen. Es geht, wie erwähnt, um die Abwehr von Empirismus und Intellektualismus ${ }^{10}$ im Zusammenhang der Frage, wie Wahrnehmung und Aufmerksamkeit zu bestimmen sind. Merleau-Ponty erörtert dabei die Verortung und Situiertheit der Dinge in der Perspektivität der Wahrnehmung. Merleau-Pontys Leibkonzeption kann nur dann in der Untersuchung des Gebetsphänomens produktiv aufgenommen werden, wenn es entsprechend als Wahrnehmung, Aufmerksamkeit bzw. als ein davon abgeleiteter oder ein zumindest ähnlicher Vor-

8 Ebd., 174.

9 Ebd., 177.

${ }^{10}$ Vgl. ebd., 47: „Beide nehmen zum Gegenstand ihrer Analyse eine objektive Welt, die weder dem Sinne noch der Zeit nach das Erste ist, beide erweisen sich als unfähig, der eigentümlichen Weise der Konstitution eines Gegenstandes im perzeptiven Bewußtsein Ausdruck zu leihen, beide wahren der Wahrnehmung gegenüber Abstand, anstatt sich auf sie einzulassen.» 
gang verstanden wird. Die Untersuchung des Gebetsphänomens, die Merleau-Pontys Anliegen folgt und sich von ihm leiten lässt, wird versuchen, das Angebetete ( $>$ Gott $\triangleleft$ ) als perspektivisch Bedingtes zu verstehen und das Gebet als perspektivische Wahrnehmung >Gottes bzw. als Aufmerksamkeit auf >Gott< zu beschreiben. Das im Gebet Angebetete ist dann bestimmt als etwas vom Anbetenden Abhängiges. Ohne den Anbetenden gibt es kein Angebetetes für den Anbetenden. Dieses Gebetsverständnis kann sich bewähren, insofern es erfahrungsmäßig evident scheint. Dabei wird das Gebet als Gewohnheit verstanden. Und die Feststellung, dass man aus Gewohnheit betet, bleibt plausibel und ihr wird nicht widersprochen.

\section{Beten aus Gewohnheit?}

Doch der Feststellung, Merleau-Pontys Leibbegriff sei zur Bestimmung des Gebetsphänomens sinnvoll, kann widersprochen werden. Die Gründe dafür, ihr eine andere Sichtweise entgegenzusetzen, ergeben sich im Rahmen des Wirklichkeitsverständnisses, das Beten als Gewohnheit versteht, nicht notwendigerweise. Auch lassen sich Gründe dabei nicht hinreichend darlegen. Intern legt sich nicht zwingend ein Widerspruch nahe. Das Motiv für den Widerspruch muss von randerswoher kommen. So kann es sich aus einem nicht rationalisierbaren Unwohlsein gegenüber den in Anspruch genommenen Vorgaben oder daran sich anschließenden Praktiken ergeben. Oder die widersprechende Konzeption übt einen positiven Reiz aus, der neugierig macht und die Begründung dafür selbst mit sich bringt. Nicht nur die Vorstellung der Perspektivität als Merkmal des Betens, sondern auch die derVerankerung des Gebets im Leib des Betenden verliert dann ihren Gehalt und bedarf der Neubestimmung.

Merleau-Pontys Leibbegriff verliert dort seine Plausibilität, wo als Grundlage des Gebets nicht mehr der menschliche Leib, sondern das im Gebet Intendierte, Gott, selbst bestimmt wird. Wenn das Gebet nicht so zu beschreiben ist, als würde mit und durch den Leib gebetet (sondern mit und durch Gott), wird dieser Leibbegriff haltlos. In einem alternativen Leibbegriff ist Gott selbst Organ und Medium des Gebets. Die theologische Tradition hat dazu die Kategorie des Geistes eingeführt. 


\section{Sich an Gott gewöhnen}

Die Meinung, das Gebet erfolge aus Gewohnheit, setzt mindestens zweierlei voraus. 1) Das Gebet sei ein Vorgang, für den der Betende (mittels seines Leibes) Voraussetzung sei. 2) Das Gebet schaffe einen Zugang zu einer Situation (`Milieu<), die sich durch bestimmte Eigenschaften und Anforderungen auszeichnet. Doch weder verantwortet und trägt das leibliche Selbst das Gebet, noch schafft Beten ein `Milieu $<$. Das Mittel des Betens, der Leib, steht nicht in der Verfügungsmacht des Betenden und das Gebet schafft nie einen auf den Betenden isolierten Bereich. Das Beten ist selbst die Voraussetzung des Betens und ist selbst die Beziehung, die es erschließt.

Mit der Vorstellung, es sei möglich, sich an Gott zu gewöhnen (insofern, als sich das Beten zu Gott im Betenden verleiblicht), steht die Überzeugung in Zusammenhang, das im Gebet Anvisierte, Gott, sei im Gebetsvorgang kooperativ. Dies folgt der Überzeugung, im Gebet gehe es um die ,Übernahmer einer gestellten Aufgabe. Die Aufgabe, sich an Gott zu gewöhnen, würde ihre Lösung entsprechend vorzeichnen und ihre Erledigung motivieren. Die persönliche Aneignung der Lösung und die nicht delegierte, sondern persönlich vollzogene Erledigung würde dabei zeigen, dass die Übernahme nicht in einem (entsprechend als blind verstandenen) Gehorsam, sondern aufgrund einer gewissen Emanzipation vom Aufgabensteller erfolgt. Der so charakterisierte Gebetsvorgang ist von der Art einer Vermittlung zweier Instanzen. Es scheint, als würde es für möglich gehalten, $>$ Gott $<$, der die Aufgabe stellt, angebetet zu werden, und den Betenden, der Gott anbeten will, durch den Vollzug des Gebets zu versöhnen. Der Betende bliebe auf das im Beten Anvisierte, ১Gott', bezogen, in der Weise, dass er diesem gerecht zu werden versucht. Das Urteil, wodurch dies dem Betenden gelingt, müsste sich der Betende selbst anmaßen. Somit gelte, dass der Betende die Kontrolle über Verlauf und Erfüllung des Gebetsvorgangs behielte. Durch Wiederholung würde sich dabei Gewohnheit einstellen. - Wird das Gebet nun jedoch anders als ein Vermittlungsvorgang bestimmt, so ist der Gebetsvorgang als der Kontrolle des Betenden ganz entzogen zu bestimmen. Wird das Gebet so bestimmt, kann sich bei ihm keine Gewohnheit einstellen. Diese Bestimmung deckt sich mit der Überzeugung, dass das Gebet nur dann Gott gilt, wenn dem Gebet auch Überraschung bis hin zum Erschüttertsein über die eigene Existenz zugrunde liegt und zugehört. Wenn man sich an >Gott 'gewöhnt, war das Gebet gar nicht auf Gott hin orientiert. 


\section{5. >Leibung< (Marion)}

Die Bestimmung des Gebets als leibliches Verstehen (und somit als Gewohnheit) ist also nicht alternativlos. Das Gebet kann auch anders bestimmt werden. Die Art der Bestimmung entscheidet sich insbesondere am Verständnis des Leibes. Wird der Leib als tragende Instanz in einem Handlungsvorgang verstanden (der Leib betet), wird das Erreichen des Handlungsziels (erfolgtes Gebet) bestimmt als die hinreichende Bedingung dafür, sich darauf zu verstehen und eine Routine zu erlangen. Wird der Leib hingegen nicht als Träger eines Vorgangs, sondern selbst als Vorgang (Leiben, ‘Leibung`) bestimmt, ist damit eine grundsätzlich andere Sichtweise verbunden. Das Ziel des Gebetsvorgangs ist dann nicht ein anvisiertes und nun erreichtes leibliches Verstehen, sondern der Gebetsvorgang ist als seine eigene Erfüllung zu bestimmen. Im und durch das Gebet wird nicht eine Gewohnheit etabliert, sondern im Gebetsvorgang kommt das Gebet $z u$ sich. Das Gebet ist nicht das Vehikel, das jemanden zu etwas hinführt, sondern der Vorgang, der das, zu dem er dient, dadurch erreicht, dass er zu ihm dient; er ist Gebet durch das Gebet. Das Gebet ist zu bestimmen alsVorgang, der zu nichts führt, sondern sich selbst genügt. Beten ist in dieser Hinsicht in anderen Zusammenhängen als dem, den es selber schafft, sinn- und zwecklos. Es kann niemandem zur Gewohnheit werden. Die Sichtweise, wonach das Gebet ein sich selbst genügender Vorgang sei, soll im Weiteren dargestellt werden. Dafür wird ein Text von Jean-Luc Marion herangezogen, der dem Leibthema gewidmet ist. Marion verbindet seine Untersuchung mit der Frage, wie das Leib- mit dem Subjektthema zusammenhängt.

\section{Selbst-Gebung des Leibes}

"Ich gebe mir nicht meinen Leib, der Leib gibt mir mich selbst. Indem ich meinen Leib erhalte, empfange ich mich selbst, ich bin ihm hingegeben " ${ }^{11}$.

In einer Auslegung einer Stelle ${ }^{12}$ bei Descartes setzt sich Jean-Luc Marion mit einem Argument auseinander, bei dem Descartes die

\footnotetext{
${ }^{11}$ Jean-Luc Marion, Leib oder das Sich-selbst-gegeben-Sein [Übers. Thomas Alferi], in: Joachim Valentin (Hg.), Wie kommt Gott in die Welt? Fremde Blicke auf den Leib Christi, Frankfurt a.M. 2009, 35-69, 62.

${ }_{12}$ René Descartes, CEuvres, hg. v. Charles Adam/Paul Tannery, Paris $1897 \mathrm{ff}$ (Nachdruck 1956ff), Bd. 7, 24-26; Bd. 9,I, 18 (übers. v. Thomas Alferi), in: Marion, Leib oder das Sich-selbst-gegeben-Sein, 37: „Bin denn nicht ich zumindest etwas? Aber ich habe doch schon verneint, irgendein Sinnesorgan oder irgendeinen Körper zu
} 
Ich-Existenz über die cogitatio ${ }^{13}$ beweist. Descartes beschäftigt sich dabei auch mit der Frage, in welchem Verhältnis das denkende Ich zu den Gegenständen der Welt steht. Marions Anliegen ist es, deutlich zu machen, dass Descartes in dieser Frage das Ich zwar nicht den dem Zweifel unterworfenen res extensae direkt zuordnet, dem Ich, das niemals dem Zweifel ausgesetzt ist, aber dennoch Körperlichkeit nicht vollständig abspricht. Obwohl dies gewöhnlich von Descartes-Auslegern anders behauptet werde, sei von Descartes das Ich durchaus als verbunden mit einem sinnlichen Körper gedacht. Die Frage, die sich dann stellt, ist, in welcher Weise denn das Ich dem Bereich der bezweifelbaren res extensae zugehören und dem Zweifel dennoch entzogen sein kann.

Die Art der Sinnlichkeit der res extensae ist von derjenigen des Ich der cogitatio jedenfalls grundlegend verschieden. Die res extensae sind fühlbar als gefühlte Körper, selbst aber nicht fühlend. Für den eigenen Körper (das Ich) hingegen ist es charakteristisch, »sich selbst $\mathrm{zu}$ fühlen und sich durch die fühlbaren Gegenstände affizieren zu lassen, gerade weil er selbst unhintergehbar fühlbar, das heißt mit Sinnlichkeit versehen ist $\aleph^{14}$. Der eigene Körper teilt mit den res extensae die Eigenschaft, fühlbar zu sein, ist aber anders als sie zusätzlich in der Lage, zu fühlen. Marion stellt fest, dass Descartes im Laufe seiner Argumentation unsachgemäß die Sinnlichkeit des cogito doch in Frage stellt und als bloß denkendes und nicht auch sinnliches Wesen bestimmt. Möglicherweise hätten Descartes die begrifflichen Mittel dazu gefehlt, das Ich als sowohl denkend als auch fühlend zu bestimmen ${ }^{15}$. Marion schlägt seinerseits vor, das hier angesprochene Phänomen »als ein Selbst, als ein Sich-Geben, erscheinen zu lassen « ${ }^{16}$.

Der eigene Körper ist also unzweifelhaft fühlend und fühlt sich. Doch er fühlt sich selbst nicht in der Weise, wie er die res extensae fühlt. Indem er im Aufgang seines Selbstdenkens sich selbst fühlt, fühlt er keinen Gegenstand (res), der ihm fremd ist, sondern eben sich selbst als Gegenstand, dem eigen ist, dass er denkt und der deshalb ein denkender Gegenstand ist (res cogitans). Das Ich denkt sich selbst.

\footnotetext{
haben. Dennoch zögere ich: Denn was folgt daraus? Bin ich so sehr vom Körper und den Sinnesorganen abhängig, daß ich ohne sie nicht sein könnte?

${ }^{13}$ René Descartes, Principia philosophiae I, \$9, in: Euvres, hg. v. Charles Adam/Paul Tannery, Paris 1897ff (Nachdruck 1956ff), Bd. 8,I, 7: „Unter dem Namen scogitatio verstehe ich alles das, was, indem wir uns dessen bewußt sind, in uns geschieht, sofern in uns ein Bewußtsein davon ist."Vgl. Claus von Bormann/Rainer Kuhlen/Ludger Oeing-Hanhoff, Art. Denken I., in: HWPh, Bd. 2, Sp. 60-102, 78.

${ }^{14}$ Leib oder das Sich-selbst-gegeben-Sein, 39.

15 Vgl. ebd., 38-42 u. 48 f.

${ }^{16}$ Ebd., 42.
} 
Das, was denkt, nennt Descartes mens ${ }^{17}$, die Seele. Und dieses denkt wiederum das, was von ihm gedacht wird (corpus et sensus). Dieses Fühlbare, was fühlend gedacht wird, geht dem Denken voraus, es ist ein `originäres Fühlen ${ }^{18}$. Am Beginn des Selbstdenkens des Ego steht nicht ein reiner Gedanke, sondern reines Fühlen.»Das Ego gibt sich als Leib - selbst wenn man dies verschleiern will «" An die Stelle des Konzepts der cogitatio tritt, so Marion (im Anschluss an Michel Henry), damit dasjenige des sursprünglichen Selbst-Fühlens‘; es meint die Affektion seiner selbst durch sich selbst ${ }^{20}$.

Durch die Auseinandersetzung mit Husserls Leibbegriff und in Aufnahme von Henrys Konzept der Selbstaffektion gelangt Marion schließlich zu seinem eigenen Leibbegriff. Dieser lautet zunächst, wie oben erwähnt: Der Leib wird nie anders als originär fühlend gefühlt. Als so fühlend ist der, der fühlt, von Anfang an mit einem Körper der Welt verbunden ${ }^{21}$. Weil er diesen »zuerst fühlt, ihn also als zu ihm gehörig fühlen und ihn sich gar zu eigen machen kann «"22. Marion stellt also zunächst eine Verbindung von Ich und Leib (als fühlbares Ich) fest und denkt schließlich beide Instanzen als eine Einheit. Das Ich kommt zu sich nicht in Verbindung mit dem Körper, sondern insofern als es selbst Leib ist; das Ich fühlt nicht seinen Körper, sondern sich selbst als Leib - "nicht anders als das Auge, das sich selbst sieht, oder der Hörsinn, der sich selbst hört ${ }^{23}$. Ich und Leib sind identisch. Das Ich nimmt keine Haltung ein, sondern ist diese Haltung. Das Ich ist, was es als Leib ist. Ausgehend von diesem Subjekt- und Leibbegriff, rekapituliert Marion Descartes' Anliegen. Er beschreibt dieses so: Descartes "führt das Ego zu seinem letzten Punkt, seinem faktischen und individuellen Selbst, seiner Leibung zurück. Und mit dieser Rückführung löst er die letztmögliche Reduktion ein: Er führt das Phänomen auf ein Gegebenes zurück, was im Fall des Ichphänomens bedeutet: auf die Leibung des Hingegebenen/Ergebenen « ${ }^{24}$.

Das Ich, als Handlungsträger und Instanz der Selbstreflexion, setzt seinen Anfang nicht selbst (indem es sich selber denkt in der cogitatio), sondern erfährt diesen Anfang an seiner eigenen Leibung. Das Ich beginnt für sich zu sein nicht durch die cogitatio, sondern in

\footnotetext{
${ }^{17}$ Vgl. ebd., 42.

${ }^{18} \mathrm{Vgl}$. ebd., 42f.

${ }^{19}$ Ebd., 43.

${ }^{20} \mathrm{Vgl}$. ebd., $48 \mathrm{f}$.

${ }^{21}$ Vgl. ebd., 45.

${ }^{22}$ Ebd., 45.

${ }^{23}$ Ebd., 49.

${ }^{24}$ Vgl. ebd., 68.
} 
der leiblichen Einsetzung als Ich in die cogitatio. Mit anderen Worten, mit Bezug auf das Gebet: Wer betet, erfährt sich nicht von selbst als Betender, sondern dadurch, dass er, zum Beten in Stand gestellt, betet. Das Konzept der Leibung beschreibt intentionales Handeln als einen Vorgang, bei dem das leibliche Fühlen dem an diesem Vorgang beteiligten Ich zuvorkommt. Bevor das Ich will, ist es als Leib bei dem, was es will.

Im Anschluss an Marions Konzept der Leibung ist das Gebet als Vorgang der Leibung zu verstehen. Dabei wird nicht beschrieben, wie ein Vorgang leiblich wird (sich als Gewohnheit verleiblicht), sondern inwiefern dieser Vorgang von Anfang an er selbst, d.h. leiblich (eine Leibung) ist.

\section{Schluss}

Für Merleau-Ponty wird im Intendieren ein Ort und eine Position (`Milieu<) eingenommen, wo man sich engagiert. Um diese Position zu erlangen, muss man sich erst in ihr einfinden und bewähren: man muss sich erst daran gewöhnen. Im Anschluss an Merleau-Ponty ist Beten als Vorgang zu bestimmen, durch den der Betende eine Position erlangt, in der er zum Beten befähigt ist. Organ und Medium des Gebets wird der Betende durch die Übung und Einübung des Gebets. Aus Gewohnheit betet er.

Für Marion hingegen ist nur im Intendieren selbst der Intendierende bestimmt als derjenige, der intendiert. Im Vorgang der >Leibung< vollzieht sich die Installation desjenigen, der intendiert. In der Leibung ist der Intendierende, derjenige, der intendiert, d.i. der Leib. $\mathrm{Zu}$ diesem Intendierenden entwickelt man sich nicht, sondern wird dazu eingesetzt. Entweder ist man eingesetzt oder nicht; eine Wahl gibt es nicht. Im Anschluss an Marions Konzept der `Leibung kann Beten deshalb wie folgt bestimmt werden: Im Beten ist der Betende Betender durch die `Betung`, d.h. den Vorgang, in dem für ihn gebetet wird, damit er beten kann. Er bestimmt sich nicht selbst zum Betenden, sondern findet sich in dieser Bestimmung vor. Was angebetet wird, wählt der Betende nicht aus, sondern ist ihm im Beten gegeben. Doch er betet nicht nur $z u$ dem, was ihm im Beten gegeben ist, sondern auch mit dem, was ihm gegeben ist. Der Betende ist selbst weder Organ noch Medium des Gebets. Sondern wer betet, ist vom Gebet eingenommen und es betet für ihn. 


\section{Das Gebet als Anzeige der Unmöglichkeit äußerer Form re- ligiöser Erfahrung}

Im Anschluss an Merleau-Pontys >Phänomenologie der Wahrnehmung` legt es sich nahe, das Gebet als ein `kulturelles Phänomen ${ }^{25}$ zu bestimmen. Entsprechend kann gesagt werden, dass das Gebet "Unwiederholbares wiederholbar ${ }^{26}$ macht. Im Beten nimmt religiöse Existenz eine Form an, die sie kultivieren kann.

Doch im Anschluss an das mit Marion gewonnene Leib- und Gebetsverständnis, ist das Gebet der Hinweis auf die Unmöglichkeit der hinreichenden Bestimmung der äußeren Form religiöser Erfahrung. Es gibt keine Form, die man übernehmen könnte und müsste. Denn, wo nicht mehr ich bete, sondern Gott betet, ist der Betvorgang nicht als Handlung bestimmt, die in >Fleisch und Blut übergeht, sondern als Handlung, deren Subjekt Gott selbst ist, dem das Gebet gilt. Wer betet, betet nicht aus (eigener) Gewohnheit, sondern aufgrund eines (gestifteten) Einwohnens in einem Gebetsvorgang. Denn im Gebet des Betenden ist nicht sein eigener Leib involviert, sondern der fremde Leib, der sich ihm für ihn hingibt. Somit geht es nicht um ein Gewöhnen an etwas oder sich Verstehen auf etwas, sondern um ein Sich-einbegriffen-Wissen in etwas. Nicht ist zu lernen, wie man selber zu >Gott betet, sondern wie man mit und durch Gott zu Gott betet. Somit ist das Beten nicht zu bestimmen als Resultat und Vorgang eines Gewöhnungsprozesses, sondern eines fortwährenden Verweilens an dem Ort, der nicht bei einem selbst, sondern bei Gott ist. Nicht bewegt sich der Betende, sondern für ihn Gott selbst zu Gott hin.

\section{Der Leib Christi}

Der Leibbegriff, der dem Leibbegriff Merleau-Pontys (Leib als leibliches Verhalten) zur Bestimmung des Gebetsphänomens entgegenzusetzen ist, konzipiert den Betenden als jemanden, der nicht für sich, aber auch nicht in Abhängigkeit von einem bestimmten Verhalten (leibliches Verhalten, Bewegung) betet, sondern in und durch die >Leibung ‘. >Leibung ist kein Vorgang, an dem Bedingendes von Bedingtem unterschieden wird, sodass sie einen Prozess beschriebe. Sondern mit `Leibung< ist die Situation beschrieben, dass das Sein und Tun bzw. Erleiden eines Subjekts nicht aufgrund eines ihnen eigenen Antriebs aufeinander bezogen sind oder sich voneinander

${ }^{25}$ Vgl. Bernhard Waldenfels, Hyperphänomene, Berlin 2012, 359.

${ }^{26}$ Ebd., 409. 
abstoßen. Sondern Tätiger und Tat sind aufgrund ihres faktischen leiblichen Aufeinanderbezogenseins aufeinander bezogen. Wird das Beten als `Leibung bestimmt, so besagt dies, dass der Betende durch das Beten betet, wobei er nicht aus eigenem Vermögen betet, sondern aufgrund der Ermöglichung und mit der Kraft, die das Beten selbst ihm gibt. Der Leib des Betens ist das, was es ermöglicht und in Gang hält. Theologisch nennt man diesen Leib den Leib für den Betenden, den `Leib Christi<, der durch den Geist Leib für den Betenden ist. Leib Christi und Geist sind ihrerseits nicht als getrennte Größen $\mathrm{zu}$ verstehen, sondern trinitätstheologisch als Erscheinungsweisen Gottes selbst zu bestimmen.

Die Bestimmung des Gebets als Gewohnheit besagt, dass durch leibliches Einstimmen des Betenden und seiner Instanzen (Leib, Wille, Bewusstsein) das Gebet eine ,Übereinstimmung zwischen Intention und Vollzug ${ }^{27}$ erreicht. Die Bestimmung des Gebets als Leibung hingegen besagt, dass mit Beten ein Vorgang beschrieben ist, der sein Wo, Womit, Wozu und Woraufhin selbst mit sich bringt.

- Marcel Egli verfasst eine Dissertation zum Thema ,Vertrauen und geschenkte Identität. Vertrauen im Anschluss an Karl Barths Versöhnungslehre.

${ }^{27}$ Merleau-Ponty, Phänomenologie der Wahrnehmung, 174: „Verstehen heißt, die Übereinstimmung erfahren zwischen Intention und Vollzug, zwischen dem, worauf wir abzielen, und dem, was gegeben ist; und der Leib ist unsere Verankerung in der Welt." 